\title{
Data Analysis on Fabrication of the Super-hydrophobic Stainless Steel Needle with Faired Properties
}

\author{
Yanling Wan, Jun Lou, Zhigang Liu, Zhongxu Lian, Huadong Yu \\ School of Mechanical and Electrical Engineering, Changchun University of Science and Technology, \\ Changchun 130022, China
}

Keywords: Stainless steel needle, Super-hydrophobic, Resistance test.

\begin{abstract}
Many creature surfaces owe to the exitance of the unusual microstructures that could influence their wet ability, these microstructures also provide a new idea for fabricating function materials. In this paper, the super-hydrophobic structures were successfully built on the surface of stainless steel needle by a simple technique combining of chemical etching and molecules modification. After the etching reaction, porous microstructures were created on the stainless steel needle surface. By modifying $1 \mathrm{H}, 1 \mathrm{H}, 2 \mathrm{H}, 2 \mathrm{H}$-perfluorodecyltriethoxysilane, the stainless steel needle showed a good super-hydrophobic property with a contact angle (CA) greater than $150^{\circ}$. Moreover, the stainless steel needle revealed obvious faired activity in resistance test. The result shows that the faired property of the super-hydrophobic needle is better than the hydrophobic one. The as-obtained needle with both super-hydrophobic and faired properties has the potential application in modern medical devices.
\end{abstract}

\section{Introduction}

In nature, many creatures reveal a variety of properties owe to the roughness on the their surfaces. This roughness generally is potentially useful to create the super-hydrophobic surface and the other function surface. Super-hydrophobic surfaces have the potential use in applications associated with self-cleaning[1] and anti-adhesive properties[2]. Up to now, various techniques were used for the fabrication of super-hydrophobic surface by electrochemical deposition[3], laser or chemical etching[4], electroless replacement deposition[5], chemical vapor deposition[6], post treating[7], so-gel processing[8], and so on. However, most of these methods are primarily suited to prepare super-hydrophobic surface on a plane surface, this methods have potential weaknesses such as requirement of using of flat two-dimensional surfaces to make a hierarchical structure. Here, a super-hydrophobic metallic surface can be easily generated, and a sample two-step fabrication process is needed in the form of chemical etching and coating.

In this paper, the needles by chemical etching and fluorination treatment with both super-hydrophobicity and faired properties were obtained successfully. The irregular microscale roughness was formed on the stainless steel needle after the etching process, which not only may effect on wettability property of the needle surface, but also the resistance of the stainless steel needle. In order to investigate the role of roughness, different etching time was prepared. 


\section{Experiment}

\section{Materials}

Needles were purchased from Jiangxi Hongda Medical Equipment Group Co. Ltd. Methanol, acetone, alcohol and 1H,1H,2H,2H-perfluorodecyltriethoxy- silane (PFDTES, 97\%), distilled water, $\mathrm{HCl}$, and $\mathrm{HF}$ were used for all experiments without any further purification.

\section{Experimental methods}

Firstly, the stainless steel needles were cleaned with distilled water successively for about $15 \mathrm{~min}$, and the cleaned needles were immersed into the solution of $\mathrm{HF}(12 \mathrm{~mol} / \mathrm{L})$ and $\mathrm{HCl}(3 \mathrm{~mol} / \mathrm{L})$, respectively. After etching, the specimens were washing in distilled water of 5 min to remove the residual solution and dried in the dying box of $80^{\circ} \mathrm{C}$ for about $20 \mathrm{~min}$. Secondly, the dried specimens were placed into the mass ratio of 1:100 mixed solutions, made up of PFDTES and methanol, for about $2 \mathrm{~h}$. Then the immersed specimens were placed in the drying box with the temperature of $135^{\circ} \mathrm{C}$ for about $2 \mathrm{~h}$ again.

\section{Characterization}

The surface morphology measurements were observed on scanning electron microscope (SEM, JMS-6700F). The contact angle (CA) were measured by the OCA20 optical contact angle measuring instrument. The average $\mathrm{CA}$ value were measured at three different positions of the same sample, and the droplet volume of water was $2 \mu \mathrm{L}$ for CA. For the resistance test, the universal tester (Shanghai hualong test instruments) was used to measure the resistance of the as-obtained stainless needles. Three samples were selected to get a average value for each test.

\section{Results and discussion}

\section{Fabrication and characterization of the stainless steel needle}

Fig. 1 shows the schematic illustration of the sample fabrication process of a stainless steel needle with the super-hydrophobicity. Here, this proposed methodology consists of the fabrication of microstructure on the stainless steel needle surface by chemical etching and fluorination treatment. In the chemical etching process, the roughness is spontaneously created in the form of the dense pits on the stainless steel needle surface. Shown in Figure 2a is SEM image of an as-etched stainless steel needle surface (Step 1: $15 \mathrm{~min}$, Step 2: $30 \mathrm{~min}$ ) with distinct scale pits. However, in Figure 2b (the obtained photos by VHX-1000 large depth 3-D scanner), the as-etched stainless steel needle surface was uniformly covered by many micro-scale mesh pits clearly. It was found that the depth of pit is $3 \mu \mathrm{m}$, and the width of pit is $20 \mu \mathrm{m}$.

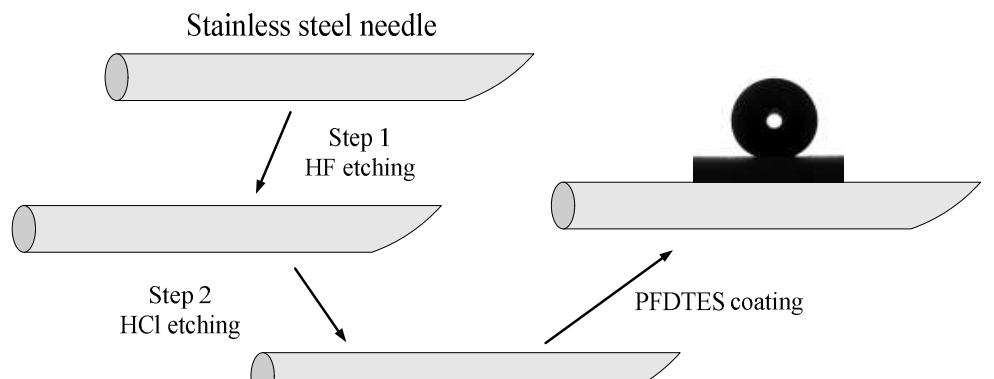

Fig. 1: Schematic illustration of the fabricated process of a stainless steel needle 

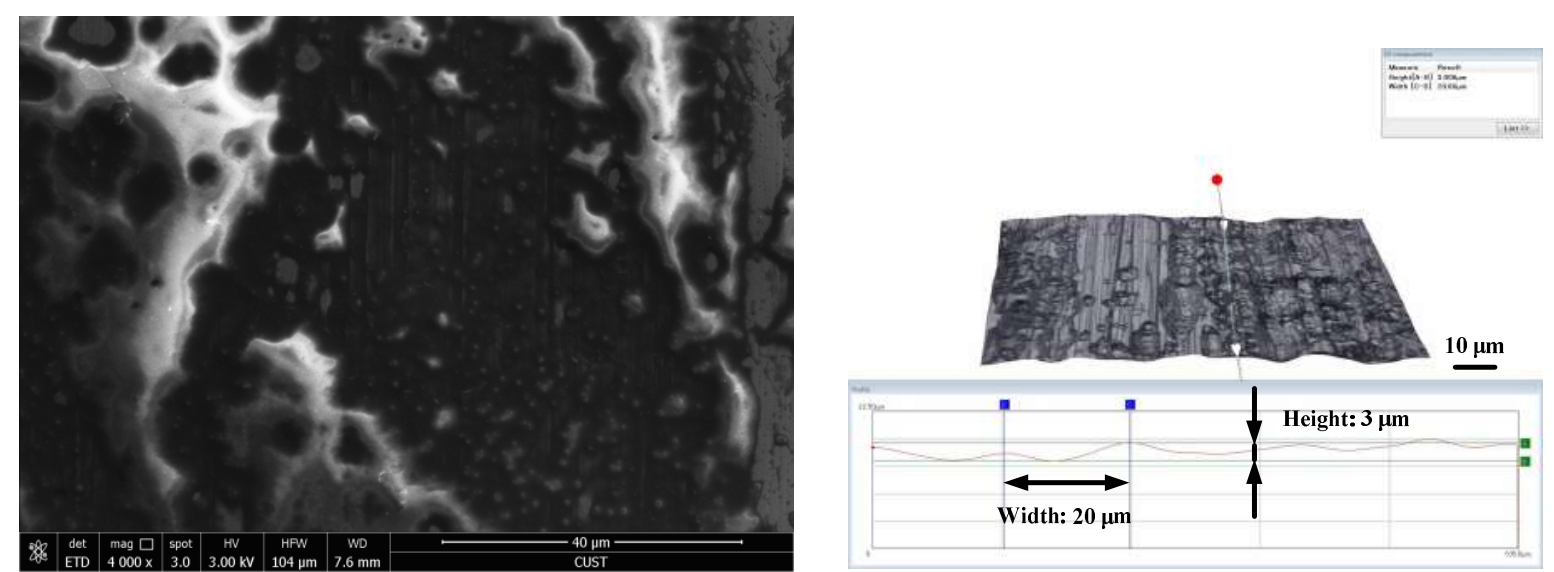

Fig.2: SEM image (a, scale bar: $40 \mu \mathrm{m}$ ) and the 2-D optical photo (b) of surface

\section{Wettability property of the stainless steel needle}

In order to investigate the effect of etching time on the wettability property of the stainless steel needle, we prepared the specimens with different etching time (Step 1: $3 \mathrm{~min}, 5 \mathrm{~min}, 10 \mathrm{~min}, 15 \mathrm{~min}$. Step 2: $10 \mathrm{~min}, 20 \mathrm{~min}, 30 \mathrm{~min}, 40 \mathrm{~min}$ ), and the contact angle (CA) experiment was preformed. After fluorination treatment, it was noted that the CA of the stainless steel needle depended on the reaction time (Figure 3). When the etching time of Step 1 increased to $10 \mathrm{~min}$ and the etching time of Step 2 increased to $30 \mathrm{~min}$ at this time, the CA value is maximum $\left(153.6^{\circ}\right)$. These results also indicated that the etching time is an important factor for preparing the microstructure of the super-hydrophobic needle surface. Besides the PFDTES coatings not only provide a super-hydrophobic needle surface, but also could modify the wettability property by changing the surface energy of the needle surface.

(a)

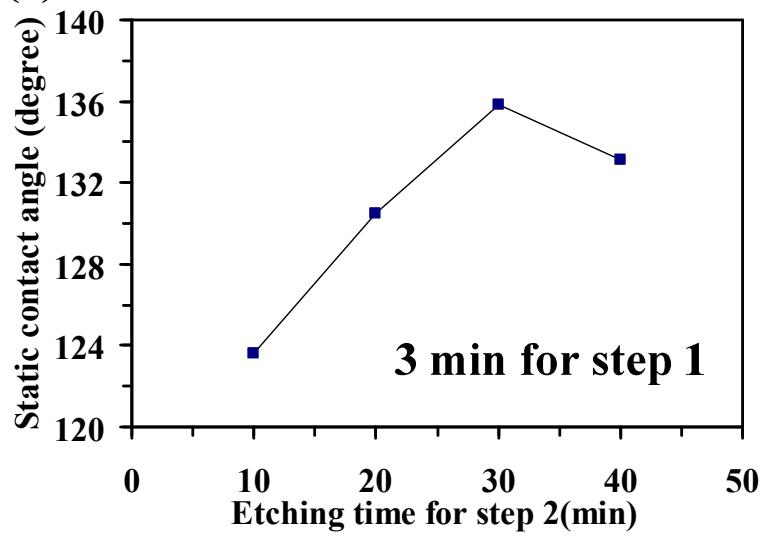

(c)

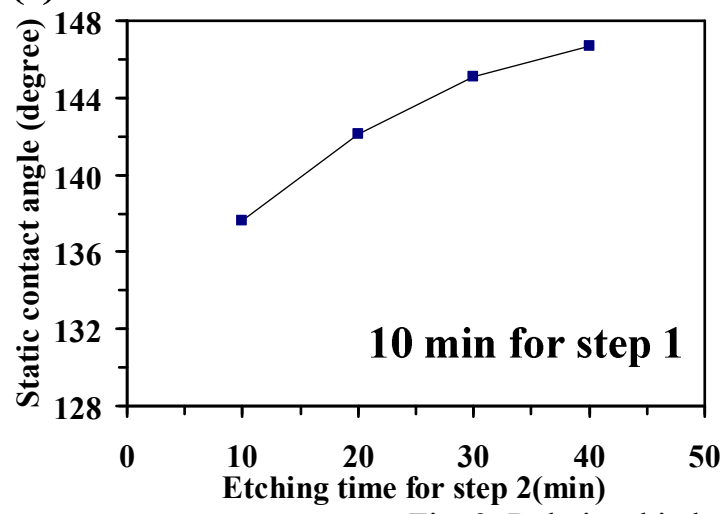

(b)

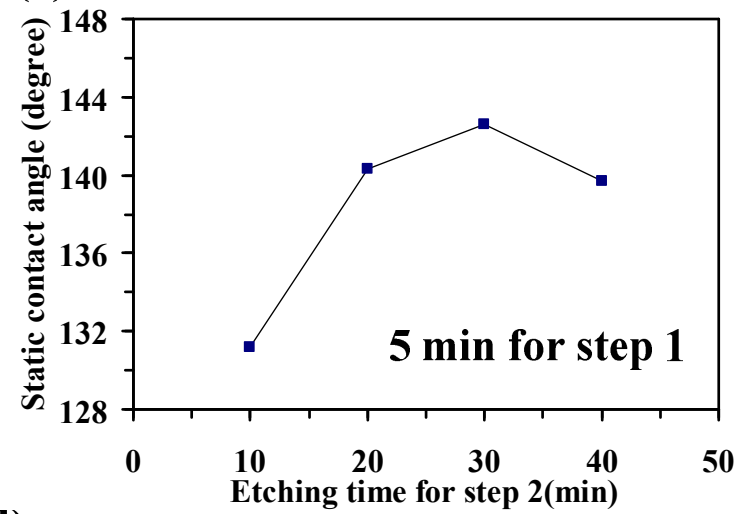

(d)

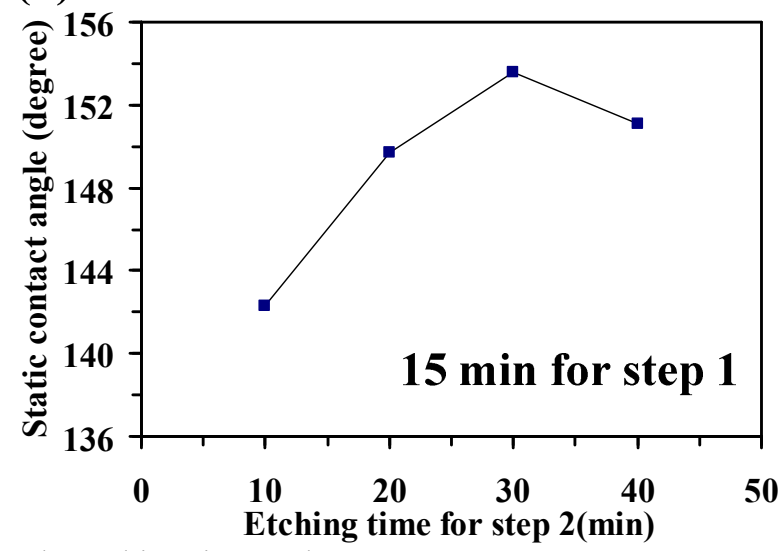

Fig. 3: Relationship between the etching time and SCA 


\section{Resistance test of the stainless steel needle}

The drag needle is important for its application in daily medical apparatus and instruments. In this paper, based on unsmooth structure drag reduction technology research, the drag reduction properties of the stainless steel needle was tested by the universal tester. The measured resistance values are shown in Figure 4. However, the etched needle revealed a smaller value than the original one $(18.3 \pm 0.1 \mathrm{~N})$. By the contrast analysis, it was also observed that faired activity was affected by the wettability property of the stainless steel needle, the faired activity of the super-hydrophobic needle is better than the hydrophobic one, that is, the resistance values decreased with the increasing of the static contact angle. The possible reason is that there were a lot of unsmooth structure on the etched needle surface.

(a)

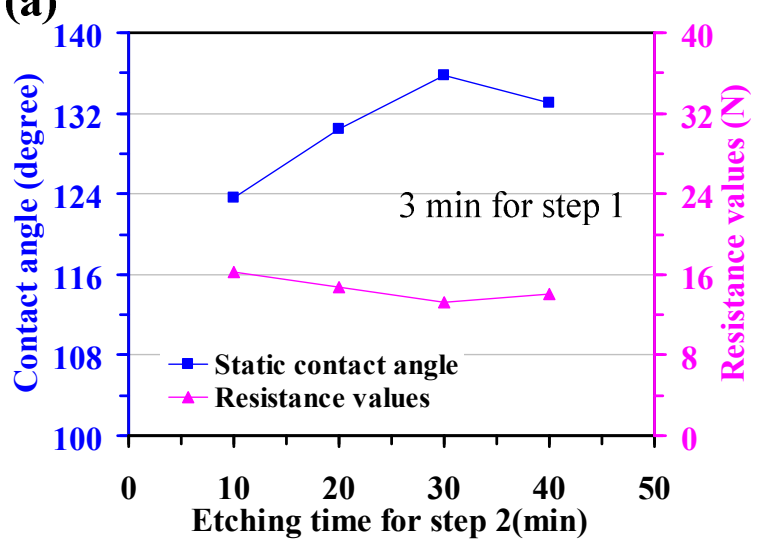

(c)

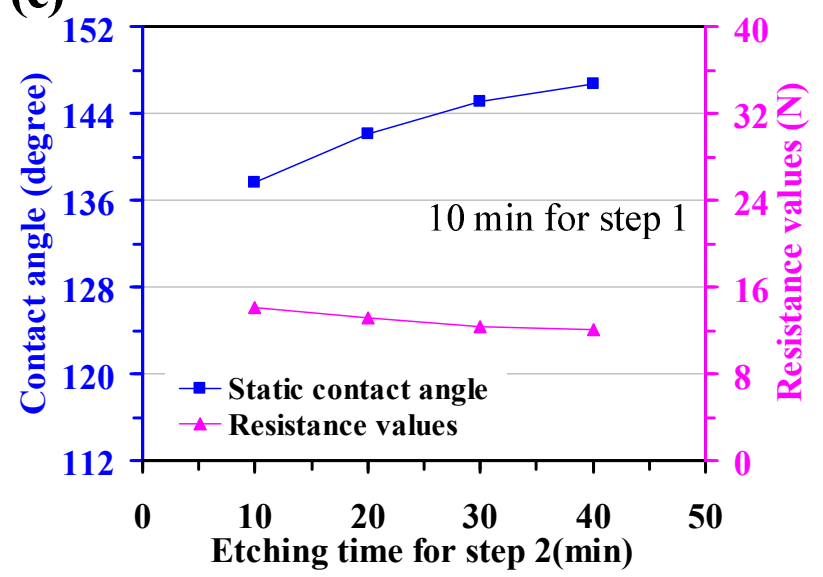

(b)

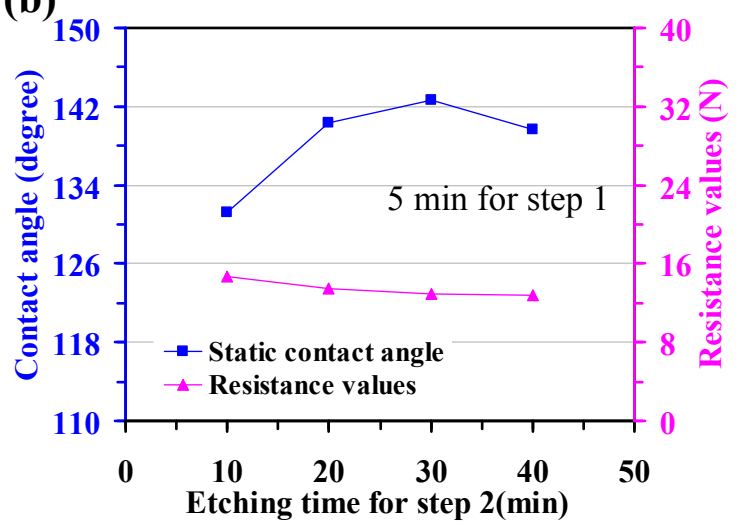

(d)

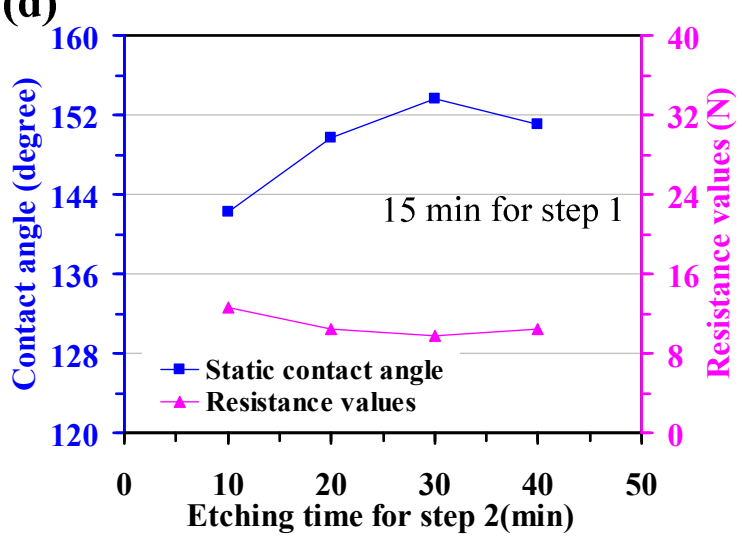

Fig. 4: Relationship between the etching time and resistance values

\section{Conclusions}

To summarize, a simple technique was demonstrated to fabricate the needle with both super-hydrophobic and faired properties. The significant super-hydrophobic stainless steel needle surface is attributed to the microstructure and low-surface-energy. Because of unsmooth microstructure, the needle has faired property. It was also found that the faired property of the super-hydrophobic needle is better than the hydrophobic one. The as-obtained needle would have the potential application in modern medical apparatus and instruments. 


\section{Acknowledgments}

This work was supported by the National Natural Science Foundation of China (51275056), Jilin Province Science and Technology Development Program of China (201201123), and the Changchun University of Science and Technology Young Scientists Foundation of China (202000490).

\section{References}

[1] Chen Y Y, Ji J G, Liu T. Preparation of a super-hydrophobic and self-cleaning coating for an anti-icing transmission line. Applied Mechanics and Materials, 2014, 543, 593-595.

[2] Jin M H, Liao M Y, Jiang L. Preparation of super-hydrophobic PDMS films and study on surface adhesion. Chemical Journal of Chinese Universities-Chinese, 2007, 28, 996-998.

[3] Zhao N, Shi F, Wang Z Q, Zhang X. Combining layer-by-layer assembly with electrodeposition of silver aggregates for fabricating super-hydrophobic surfaces. Langmuir, 2005, 21, 4713-4716.

[4] Chen W, Fadeev A Y, Heieh M C, Öner D, Youngblood J, McCarthy T J. Ultrahydrophobic and ultralyophobic surfaces: some comments and examples. Langmuir, 1999, 15, 3395-3399.

[5] Song W, Zhang J J, Xie Y F, Cong Q, Zhao B. Large-area unmodified super-hydrophobic copper substrate can be prepared by an electroless replacement deposition. Journal of Colloid and Interface Science, 2009, 329, 208-211.

[6] Li H, Wang X, Song Y, Liu Y, Li Q, Jiang L, Zhu D. Superamphiphobic aligned carbon nanotube films. Angewandte Chemie, 2001, 113, 1793-1796.

[7] Khorasani M T, Mirzadeh H, Kermani Z. Wettability of porous polydimethylsiloxane surface: morphology study. Applied Surface Science, 2005, 242, 339-345.

[8] Nakajima A, Fujishima A, Hashimoto K, Watanabe T. Preparation of transparent superhydrophobic boehmite and silica films by sublimation of aluminum acetylacetonate. Advanced Materials, 1999, 11, 1365-1638. 